\title{
There is no significant correlation of adenomyosis with benign, premalignant and malignant gynecological pathologies. Retrospective study on 647 specimens
}

\author{
Michail Matalliotakis ${ }^{1}{ }^{(1)}$, Maria I. Zervou ${ }^{2}$, Charoula Matalliotaki ${ }^{3}$, George N. Goulielmos ${ }^{4}$, \\ Konstantinos Krithinakis ${ }^{3}$, Georgios Kapetanios ${ }^{1}$, Ioannis Kalogiannidis ${ }^{1}$ \\ ${ }^{1} 3^{\text {rd }}$ Department of Obstetrics and Gynecology, Aristotle University of Thessaloniki, Konstantinoupoleos, Thessaloniki, Greece \\ ${ }^{2}$ Section of Molecular Pathology and Human Genetics, Department of Internal Medicine, School of Medicine, \\ University of Crete, Heraklion, Greece \\ ${ }^{3}$ Department of Obstetrics and Gynaecology, Venizeleio and Pananio General Hospital of Heraklion, Heraklion, Greece \\ ${ }^{4}$ Section of Molecular Pathology and Human Genetics, Department of Internal Medicine, School of Medicine, \\ University of Crete, Heraklion, Greece
}

\begin{abstract}
Objectives: The aim of this study is to identify the prevalence of benign, premalignant and malignant gynecological pathologies in women with adenomyosis who underwent gynecological surgery.

Material and methods: The medical records collected between 1985 and 2020 were retrospectively reviewed. The pathology reports were studied from 647 cases where adenomyosis was presented. The estimated prevalence of benign, premalignant and malignant gynecological disorders in the general population was further evaluated.

Results: The mean age of women with adenomyosis was $54.1 \pm 10.4$ years old. Out of 647 patients, in $18.5 \%$ of the specimens we detected isolated adenomyosis and in $81.5 \%$ of cases a coexistence of one or more gynecological diseases, while in 84 out of 647 patients (13\%) there was coexistence of adenomyosis with more than one gynecological condition (benign or malignancy). Among all cases, uterine leiomyomas were observed in $61.3 \%$ of patients, followed by endometrial polyps (11.9\%), endometriosis (11.6\%), endometrial hyperplasia (7.1\%), endometrial cancer (3.6\%), ovarian (1.4\%) and cervical cancer $(0.8 \%)(p<0.001)$.Additionally, we found that women with a simultaneous co-existence of adenomyosis, leiomyomas and endometrial polyps or hyperplasia were younger $(p<0.01)$ in comparison to cases with malignancy.

Conclusions: Adenomyosis presents a common benign but often progressing myometrial condition that it is underestimated in clinical practice. Even though some studies suggest a potential association with several gynecological pathologies, we did not confirm a significant difference of adenomyosis prevalence between benign, premalignant and malignant gynecological conditions compared with the general population. Further investigation is required to confirm our results. Key words: adenomyosis; endometriosis; benign gynecological diseases; gynecological malignancy
\end{abstract}

Ginekologia Polska 2022; 93, 6: 467-472

\section{INTRODUCTION}

Adenomyosis is an enigmatic benign myometrial lesion characterized by the overgrowth of ectopic endometrium, into myometrium layers, resulting in a diffusely or focally enlarged uterus [1]. In 1860, Carl von Rokitansky was the first who described this entity as "cystosarcoma adenoids uterinum" [2].
With prevalence ranges reported between 10-70\%, it is detected more often in women between 40-55 years old. Vercellini et al. [3], postulated that adenomyosis can be observed in $30-60 \%$ of resected uterine specimens.

According to the literature, it can coexist with endometriosis lesions and myomas cases. Although being different entities, $35-55 \%$ of women diagnosed with myomas 
share similar clinical characteristics to adenomyosis such as abnormal vaginal bleeding, painful menstrual cycles and chronic pelvic pain. Additionally, there have been reports of a coincidence with endometrial hyperplasia, polyps and endometrial cancer [4-7].

The risk factors for adenomyosis include age in the mid-forties, multiparity, smoking habits and elevated hormonal levels such as FSH and prolactin. Moreover, prior cesarean section and uterine surgery represent a major risk due to their potential to damage the junctional zone [2].

\section{Objectives}

The design of this study aims to investigate and highlight a possible notable relationship between adenomyosis and benign, premalignant and malignant gynecological diseases in women underwent gynecological surgery.

\section{MATERIAL AND METHODS}

\section{Characteristics of study population}

We reviewed the medical records of patients that underwent gynecological surgery between 1985 and 2020, from the department of Obstetrics and Gynecology of the Venizeleio General Hospital of Heraklion and between 2009 and 2020 from the $3^{\text {rd }}$ Department of Obstetrics and Gynecology of Aristotle University of Thessaloniki. A total of 647 women with adenomyosis, which were confirmed by histology were retrospectively examined. Data were recorded including age, the type and cause of surgery performed and the concurrency of other benign and malignant gynecological conditions. We excluded cases without histological evidence. The pathologic features of the gynecologic diseases were classified according to the criteria of FIGO [8]. The Ethics Committee of the Department of Obstetrics and Gynecology of Venizeleio Hospital of Crete and the $3^{\text {rd }}$ Department of Obstetrics and Gynecology of Aristotle University of Thessaloniki approved the protocol. The data that support the findings of this study are available from the corresponding author upon reasonable request.

\section{Statistical analysis}

Student- $t$ test and $x^{2}$ tests were used for comparison of the mean of the various characteristics. The Mann-Whitney $U$ test was performed if data were not distributed normally. The results are reported as mean \pm SD or as percentages where appropriate. Differences were considered statically significant at $p<0.05$.

\section{RESULTS}

In the present study we included patients with a diagnosis of adenomyosis based on histopathological results from uterine-sparing and non-uterine-sparing speci-

\begin{tabular}{|c|c|}
\hline Gynecological conditions & No \\
\hline Total number of cases with coexisted pathology & $84 / 647(13 \%)$ \\
\hline $\begin{array}{l}\text { Coexistence with leiomyomas and endometrial } \\
\text { polyps }\end{array}$ & 43 \\
\hline $\begin{array}{l}\text { Coexistence with leiomyomas and endometrial } \\
\text { hyperplasia }\end{array}$ & 27 \\
\hline $\begin{array}{l}\text { Coexistence with complex hyperplasia with atypia, } \\
\text { endometrial polyps and leiomyomas }\end{array}$ & 1 \\
\hline $\begin{array}{l}\text { Coexistence with ovarian serous cystadenoma, } \\
\text { leiomyomas and endometrial polyps }\end{array}$ & 1 \\
\hline $\begin{array}{l}\text { Coexistence with ovarian serous cystadenofibroma } \\
\text { and leiomyomas }\end{array}$ & 1 \\
\hline $\begin{array}{l}\text { Coexistence with ovarian serous cystadenoma and } \\
\text { endometrial polyps }\end{array}$ & 1 \\
\hline $\begin{array}{l}\text { Coexistence with ovarian serous-mucinous cyst and } \\
\text { endometriosis }\end{array}$ & 1 \\
\hline Coexistence with ovarian fibroma and leiomyomas & 2 \\
\hline $\begin{array}{l}\text { Coexistence with ovarian fibroma, endometrial } \\
\text { polyps and leiomyomas }\end{array}$ & 1 \\
\hline $\begin{array}{l}\text { Coexistence with ovarian mucinous adenofibroma } \\
\text { and leiomyomas }\end{array}$ & 1 \\
\hline $\begin{array}{l}\text { Coexistence with brenner tumor and serous } \\
\text { cystadenoma }\end{array}$ & 1 \\
\hline Coexistence with cini and leiomyomas & 1 \\
\hline Coexistence with cervical cancer and endometriosis & 1 \\
\hline Coexistence with breast cancer and leiomyomas & 1 \\
\hline Coexistence with endometriosis and leiomyomas & 1 \\
\hline
\end{tabular}

Number of cases (No), percentage (\%). This table describes the frequency of occurrence of malignant gynecological pathologies in 647 women with adenomyosis

mens. A total of 647 women with adenomyosis were identified with or without other gynecological pathology. We detected 120 (18.5\%) cases with isolated adenomyosis and 527 (81.5\%) cases with coexistence of one or more gynecological pathologies. Furthermore, we reported the coincidence of adenomyosis with more than one gynecological disease in 13\% (84/647) of the overall cases of patients with adenomyosis (Tab. 1).

The mean age of patients was $54.1 \pm 10.4$ years old (at the time of operation). The main indications for operation were uterine bleeding, benign and malignant gynecological pathology of the genital tract and/or pelvic pain. Out of all cases, uterine leiomyoma was reported in $61.3 \%$ (397/647) of cases, followed by endometrial polyps ( $11.9 \%$ [77/647]), endometriosis (11.6\% [75/647]) and endometrial hyperplasia (7.1\% [46/647]) ( $p<0.001)$ (Tab. 2).

Table 3 shows the co-existence of adenomyosis with gynecological malignancies. Among them, $3.6 \%(23 / 647)$ had endometrial cancer, $1.4 \%(9 / 647)$ ovarian and $0.8 \%$ (5/647) cervical, respectively. 


\begin{tabular}{|l|c|}
\hline $\begin{array}{l}\text { Table 2. Histological confirmation of benign and premalignat } \\
\text { gynecological pathology in } 647 \text { women with adenomyosis }\end{array}$ \\
\hline Gynecological pathologies & No (\%) \\
\hline Uterine Leiomyomas & $397(61.3 \%)$ \\
\hline Endometriosis & $75(11.6 \%)$ \\
\hline Endometrial polyps & $77(11.9 \%)$ \\
\hline Endometrial hyperplasia & $46(7.1 \%)$ \\
\hline Endometrial hyperplasia with atypia & $10(1.5 \%)$ \\
\hline Benign ovarian cyst & $22(3.4 \%)$ \\
\hline Ovarian fibroma-thecoma & $1(0.15 \%)$ \\
\hline Ovarian fibroma & $4(0.6 \%)$ \\
\hline Ovarian mucinous cystadenofibroma & $1(0.15 \%)$ \\
\hline Ovarian serous cystadenoma & $18(2.8 \%)$ \\
\hline Ovarian mucinous cystadenoma & $1(0.15 \%)$ \\
\hline Ovarian mucinous adenofibroma & $1(0.15 \%)$ \\
\hline Brenner tumor & $1(0.15 \%)$ \\
\hline Ovarian mucinous cystadenoma (borderline) & $1(0.15 \%)$ \\
\hline CIN (cervical intraepithelial neoplasia) & $7(1.1 \%)$ \\
\hline
\end{tabular}

Number of cases (No), percentage (\%). This table describes the frequency of occurrence of benign and premalignant gynecological pathologies in 647 women with adenomyosis. Note that the sum of all gynecological conditions exceeds the total number of cases, because of the occurrence of coexisting pathologies

Table 3. Coexistence of gynecological cancer and adenomyosis

\begin{tabular}{|l|c|}
\hline Gynecological malignancy & No (\%) \\
\hline Endometrial cancer & $23(3.6 \%)$ \\
\hline Cervical cancer & $5(0.8 \%)$ \\
\hline $\begin{array}{l}\text { Stump (uterine smooth muscle tumor of } \\
\text { uncertain malignant potential) }\end{array}$ & $1(0.15 \%)$ \\
\hline Ovarian cancer & $9(1.4 \%)$ \\
\hline
\end{tabular}

Number of cases (No), percentage (\%). This table describes the frequency of occurrence of malignant gynecological pathologies in 647 women with adenomyosis

Additionally, we observed that benign lesions were more prevalent in younger ages $(p<0.01)$. Diversely, endometrial and ovarian cancers were prominent in postmenopausal women.

\section{DISCUSSION}

Adenomyosis has become a clinical challenge rather than solely a histological diagnosis. Although it has been correlated with various gynecological pathologies, in the present study, we did not confirm a significant difference of adenomyosis prevalence in benign, premalignant and malignant cases compared to the general population.

It has been shown previously that the gynecological benign tumors may develop as a result of inflammatory, dietary, genetic and environmental factors [9]. In 1972, Bird defined adenomyosis as a chronic benign invasion of endometrium (glands and stroma) into myometrium, producing an enlarged uterus that is characterized by hyperplasia and hypertrophy of tissue [10]. Even though, the exact pathophysiology of adenomyosis is not clear yet, the classical theory attributes to the disruption of the boundary between endometrial basalis layer and the underline myometrium. Moreover, the de novo metaplasia of embryonic or adult stem cells in the myometrium and the altered lymphatic drainage present well accepted alternative pathogenic theories. In recent years, a notable number of studies have shown that various inflammatory molecules, sex steroid hormone receptors, extracellular matrix enzymes, growth and neuroangiogenic factors play an important role in the pathogenesis of this condition [1]. Considering the multifactorial character of the pathophysiology of adenomyosis, genetic alternations in signaling pathways also interact in the manifestations of such patients. In parallel, it is well known that endometriosis arises from the interplay between environmental and genetic factors. Thus, many studies aimed to the identification of potential shared genetic factors involved in the development of adenomyosis, endometriosis, uterine leiomyoma and endometrial polyps. Whilst the difference observed in the expression levels of estrogen receptor (ER) and progesterone receptor (PR) between patients with adenomyosis and leiomyoma [11], significantly elevated levels of metalloproteinases (MMPs) MMP-2 and MMP-9 and some specific cytokines have been found in patients with adenomyosis, leiomyoma and endometrial polyps [12].

An oestrogen receptor-alpha ( $E R a)$ Pvull gene polymorphism was also found to be associated with endometriosis, adenomyosis and leiomyoma [13]. On the other hand, genetic polymorphisms of MMP-1, MMP-7 and peroxisome proliferator activated receptor $Y$ (PPAR $\gamma$ ) genes have been characterized as risk factors for adenomyosis and endometriosis but not for leiomyoma [14]. A recent next generation sequencing (NGS)-based study showed that the presence of KRAS mutations in adenomyosis is associated with the co-occurrence of endometriosis [15]. Of note, polymorphisms of two angiogenic factors, namely fibroblast growth factor (FGF) -1 and -2 may be involved in the initiation of angiogenesis in endometriosis and adenomyosis [16]. In sum, the aforementioned findings point out that the co-existence of adenomyosis with other gynecological conditions can be attributed to some shared genetic risk factors; obviously, there are various additional genes that are disease-specific.

Of note, four parameters describe adenomyosis. The presence of endometrial glands and stroma within the myometrium, the depth of penetration, the extend of spread in terms of number of foci and the arrangement of the lesion [2]. In last decades, although adenomyosis is 
rarely diagnosed before hysterectomy, the improvement of diagnostic approaches such as Ultrasonography and Magnetic resonance imaging (MRI) allows us to identify the disease by means of non-invasive methods. Common transvaginal ultrasound (TVUS) findings represent reliable criteria to strongly suggest the diagnosis of adenomyosis. Additionally, minimally invasive techniques such as sonography, hysteroscopy and laparoscopy can also be useful for imaging [2].

As far as the clinical signs and symptoms are concerned, patients can be asymptomatic or nonspecific features can be present like dysmenorrhea, dyspareunia, chronic pelvic pain, abnormal uterine bleeding and infertility. These overlapping symptoms can also be a manifestation of other concomitant gynecologic disorders; thus, adenomyosis has largely been a postoperative diagnosis made by the pathologist [2].

More in depth, previous studies have suggested that a co-morbidity associating exists between adenomyosis and benign and malignant gynecological tumors [4-7, 17]. Results from a large retrospective study on patients undergoing hysterectomy showed that women with leiomyoma and adenomyosis were more likely to report similar complaints [18]. Despite the hyper estrogenic environment that may share these gynecological conditions [19], in the present study, we noticed a quite expected percentage of adenomyosis coexisting with leiomyoma cases (61.3\%), followed by endometrial polyps (11.9\%), endometriosis (11.6\%) and endometrial hyperplasia (7.1\%). By taking into consideration the epidemiological standards of current literature related to earlier mentioned benign pathologies in the general population, we did not notice a prominent appearance of such conditions in women diagnosed with adenomyosis. More specific, it is already known that up to $70 \%$ of women develop leiomyoma by the age of menopause [20]. Furthermore, depending on the different geographical areas and population studied, the prevalence of endometrial polyps varies from $7.8 \%$ to almost $35 \%$ [21]. Apart from that, $10 \%$ of premenopausal and $6 \%$ of menopausal women report the coexistence of an abnormal uterine bleeding and endometrial hyperplasia at some point during their life time [22].

In association with endometriosis, from a pathogenic standpoint, they are considered two distinct diseases because of specific causative pathways and clinical presentation, despite appearing together in patients; although for over 90 years endometriosis and adenomyosis were considered as the same entity with the exception of endometriomas $[1,19]$. Worth of note, in general community endometriosis affects 10 to $15 \%$ of patients of reproductive age [23]. In the present work, we confirmed the concurrence of adenomyosis with 75 endometriosis cases (11.6\%).
Interestingly, as reported in the literature, adenomyosis may be the precursor of cancerous transformation due to the pattern of growth, invasion and angiogenesis of such lesions [24]. Well of note, malignant transformation of adenomyosis is more observed in postmenopausal and elderly women and in preexisting benign gynecological lesions [7]. Malignant changes in adenomyosis have been reported in $6.8 \%$ of patients with endometrial cancer [25]. Recently, it was postulated that women with adenomyosis are at higher risk of endometrial and thyroid cancer, while cases with endometriosis are in danger of endometrial and ovarian cancers $[17,19]$. Even thought, the association of adenomyosis in the pathogenesis of endometrial cancer is unclear, endometrial cancer involving adenomyosis is influenced by hormonal imbalances and it is associated with a better outcome $[7,19]$. Additionally, the malignant transformation of adenomyosis is suggested to be due to its endometrial epithelium transition to cancerous cells, which results to tumorigenesis [7].

Lately, Koray et al., determined the effects of adenomyosis on the aggressiveness of endometrial cancer. Although, adenomyosis was not noticed to be an independent prognostic factor for endometrial tumor, they detected a better prognosis on such cases [26]. On the other hand, in a large pilot study, the authors did not find any significant difference between patients with coexistence of endometrial cancer and adenomyosis in comparison to isolated endometrial cancer cases in terms of epidemiologic, clinopathologic and prognostic characteristics [27]. Moreover, recently, Raffone A et al., in a systematic review and meta-analysis confirmed that adenomyosis and endometrial cancer pathology present two unrelated conditions. In that study they assessed the prevalence of adenomyosis in women with endometrial cancer. They did not detect a difference in adenomyosis prevalence in endometrial cancer cases compared to those reported for other gynecological pathologies requiring surgery [28]. Furthermore, it has be revealed that the presence of adenomyosis may be a principal factor for the determination of adenocarcinoma prognosis, due to de novo malignant transformation of adenomyotic foci and the simultaneous malignant transformations in the eutopic endometrium and adenomyosis. In a previous study, Koshiyama et al., reported four cases with adenocarcinoma out of 564 women with adenomyosis $[19,29]$. Thus, a possibility of increased risk for endometrial cancer in patients with adenomyosis is yet to be confirmed.

As far as the ovarian cancer is concerned, Shen et al. [30], in a large case control study confirmed that adenomyosis importantly raise the risk of developing ovarian cancer. Opposed to that, Jeh et al., reported that adenomyosis did not increase the risk for ovarian cancer [17]. Even 
though, it is known that adenomyosis and ovarian cancer are characterized by estrogen disturbance and menstrual disorders, further investigation is required in order to establish a correlation between these two entities. In our series, we confirmed that endometrial cancer appears in $3.6 \%$ of adenomyosis patients, followed by ovarian cancer (1.4\%) and cervical cancer (0.8\%) (Tab. 3). Well of note, the National Cancer Institute mentions that approximately $3.1 \%$ of women will be diagnosed with uterine cancer, less than $2 \%$ with ovarian cancer and $0,6 \%$ with cervical cancer at some point during their lifetime [31]. Thus, according to our data, we did not observe a significant risk of developing gynecological malignancy in women with adenomyosis compared to cases with no previous pathology of adenomyosis.

On a gene level, although adenomyosis can be a precursor of some carcinomas, the exact molecular mechanisms leading to the malignant transformation are poorly understood, taking into account that studies in this field reporting genetic alterations, epigenetic changes and mutational analysis are very few. Therefore, to date, there have not been accumulated sufficient genetic and epigenetic data for the mechanisms leading to the malignant transformation of adenomyosis through a multi-step process. Both adenomyosis and (type 1) endometrial cancer have been linked to sex steroid action, while the gene expression profile of this condition is reminiscent of cancer, cell death and cell cycle networks [32]. Furthermore, reduced levels of mRNA of Phosphatase and Tensin Homolog (PTEN mRNA) were observed in adenomyosis and it is worth noting that respective gene has been characterized as a tumor suppressor gene that is mutated in a large number of cancers [33]. The relationship between PTEN and endometrial cancer has been sufficiently documented [34], while two more features strengthening the speculation for a possible link between adenomyosis and endometrial cancer deals with the implication of KRAS and BCL2 gene mutations [32]. A meta-analysis of two genome wide association studies (GWAS) of patients with uterine leiomyomas revealed shared signals with endometriosis, including two variants at the GREB1 gene as well as the rs2202282 variant of SULT1E1 genethatislocatedinacluster of sulfotransferasegenes and the rs765333492 variant of SCFD2 gene; substantially, this study presented evidence for a shared risk factor between leiomyoma and endometrial cancer (EC), rs10917151 variant of CDC42/WNT4 locus [35]. However, no association with adenomyosis was detected.

The retrospective nature of the study represents a limitation of this work. Additionally, the prevalence of adenomyosis is slightly deviated due to the postmenopausal age at the time of operation. On the other hand, strong points are the large number of patients and the selection of all cases based on histological biopsy.

\section{CONCLUSIONS}

To summarize, in the present retrospective study, the supposed association between adenomyosis and benign, premalignant and malignant gynecological diseases appears unsupported. Compared to women with no previous pathology of adenomyosis, we did not report a significant correlation of adenomyosis and other gynecological pathologies. Further studies are required in order to confirm our findings.

\section{Acknowledgment}

We would like to thank Professor loannis Matalliotakis and all the clinicians and pathologists for providing the data used in this study.

\section{Ethical approval}

The Ethics Committee of the Department of Obstetrics and Gynecology of Venizeleio Hospital of Crete (no. 124/17/2019) and the $3^{\text {rd }}$ Department of Obstetrics and Gynecology of Aristotle University of Thessaloniki [no.94/23-4-20] approved the protocol. This article does not contain any studies with animals performed by any of the authors.

\section{Informed Consent}

Informed consent was obtained from all individual participants included in the study.

\section{Conflict of interest}

The authors report no conflict of interest.

\section{REFERENCES}

1. Guo SW. The Pathogenesis of adenomyosis vis-à-vis endometriosis. J Clin Med. 2020; 9(2), doi: 10.3390/jcm9020485, indexed in Pubmed: 32050720.

2. Matalliotakis IM, Kourtis Al, Panidis DK. Adenomyosis. Obstet Gynecol Clin North Am. 2003; 30(1): 63-82, viii, doi: 10.1016/s08898545(02)00053-0, indexed in Pubmed: 12699258.

3. Vercellini P, Viganò P, Somigliana E, et al. Adenomyosis: epidemiological factors. Best Pract Res Clin Obstet Gynaecol. 2006; 20(4): 465-477, doi: 10.1016/j.bpobgyn.2006.01.017, indexed in Pubmed: 16563868.

4. Filip G, Balzano A, Cagnacci A. Histological evaluation of the prevalence of adenomyosis, myomas and of their concomitance. Minerva Ginecol. 2019; 71(3): 177-181, doi: 10.23736/S0026-4784.18.04291-0, indexed in Pubmed: 30486633.

5. Munro MG. Uterine polyps, adenomyosis, leiomyomas, and endometrial receptivity. Fertil Steril. 2019; 111(4): 629-640, doi: 10.1016/j.fertnstert.2019.02.008, indexed in Pubmed: 30929720.

6. De Wilde RL, Wallwiener M, Di Spiezio Sardo A, et al. Adenomyosis and myomata: risks, problems, and complications in diagnosis and therapy of adenomyosis and myomata. Biomed Res Int. 2018; 2018: 5952460, doi: 10.1155/2018/5952460, indexed in Pubmed: 30175136.

7. Habiba M, Pluchino N, Petignat $P$, et al. Adenomyosis and endometrial cancer: literature review. Gynecol Obstet Invest. 2018; 83(4): 313-328, doi: 10.1159/000487320, indexed in Pubmed: 29874641.

8. Gassman WT. Announcements Figo Stages- 1988 Revision. GynecolOncol. 1989; 35: 125-127.

9. Mahnert N, Morgan D, Campbell D, et al. Unexpected gynecologic malignancy diagnosed after hysterectomy performed for benign indications. Obstet Gynecol. 2015; 125(2): 397-405, doi: 10.1097/AOG.0000000000000642, indexed in Pubmed: 25569001. 
10. Bird CC, McElin TW, Manalo-Estrella P. The elusive adenomyosis of the uterus--revisited. Am J Obstet Gynecol. 1972; 112(5): 583-593, doi: 10.1016/0002-9378(72)90781-8, indexed in Pubmed: 5059589.

11. Mehasseb MK, Panchal R, Taylor AH, et al. Estrogen and progesterone receptor isoform distribution through the menstrual cycle in uteri with and without adenomyosis. Fertil Steril. 2011; 95(7): 2228-35, 2235.e1, doi: 10.1016/j.fertnstert.2011.02.051, indexed in Pubmed: 21444077.

12. Inagaki $\mathrm{N}$, Ung L, Otani T, et al. Uterine cavity matrix metalloproteinases and cytokines in patients with leiomyoma, adenomyosis or endometrial polyp. Eur J Obstet Gynecol Reprod Biol. 2003; 111(2): 197-203, doi: 10.1016/s0301-2115(03)00244-6, indexed in Pubmed: 14597251.

13. Kitawaki J, Obayashi $\mathrm{H}$, Ishihara $\mathrm{H}$, et al. Oestrogen receptor-alpha gene polymorphism is associated with endometriosis, adenomyosis and leiomyomata. Hum Reprod. 2001; 16(1): 51-55, doi: 10.1093/humrep/16.1.51, indexed in Pubmed: 11139535.

14. Shan K, Lian-Fu Z, Hui Du, et al. Polymorphisms in the promoter regions of the matrix metalloproteinases-7, -9 and the risk of endometriosis and adenomyosis in China. Mol Hum Reprod. 2006; 12(1): 35-39, doi: 10.1093/molehr/gal002, indexed in Pubmed: 16455621.

15. Inoue $S$, Hirota $Y$, Ueno T, et al. Uterine adenomyosis is an oligoclonal disorder associated with KRAS mutations. Nat Commun. 2019; 10(1): 5785, doi: 10.1038/s41467-019-13708-y, indexed in Pubmed: 31857578.

16. Kang S, Li SZ, Wang Na, et al. Association between genetic polymorphisms in fibroblast growth factor (FGF)1 and FGF2 and risk of endometriosis and adenomyosis in Chinese women. Hum Reprod. 2010; 25(7): 1806-1811, doi: 10.1093/humrep/deq128, indexed in Pubmed: 20504870.

17. Yeh CC, Su FH, Tzeng CR, et al. Women with adenomyosis are at higher risks of endometrial and thyroid cancers: A population-based historical cohort study. PLOS ONE. 2018; 13(3): e0194011, doi: 10.1371/journal. pone.0194011.

18. Taran FA, Wallwiener $M$, Kabashi $D$, et al. Clinical characteristics indicating adenomyosis at the time of hysterectomy: a retrospective study in 291 patients. Arch Gynecol Obstet. 2012; 285(6): 1571-1576, doi: 10.1007/s00404-011-2180-7, indexed in Pubmed: 22193824.

19. Verit FF, Yucel O. Endometriosis, leiomyoma and adenomyosis: the risk of gynecologic malignancy. Asian Pac J Cancer Prev. 2013; 14(10): 5589-5597, doi: 10.7314/apjcp.2013.14.10.5589, indexed in Pubmed: 24289548.

20. Stewart EA, Cookson CL, Gandolfo RA, et al. Epidemiology of uterine fibroids: a systematic review. BJOG. 2017; 124(10): 1501-1512, doi: 10.1111/1471-0528.14640, indexed in Pubmed: 28296146.

21. American Association of Gynecologic Laparoscopists. AAGL practice report: practice guidelines for the diagnosis and management of endometrial polyps. J Minim Invasive Gynecol. 2012; 19(1): 3-10, doi: 10.1016/j.jmig.2011.09.003, indexed in Pubmed: 22196255.

22. Sobczuk K, Sobczuk A. New classification system of endometrial hyperplasia WHO 2014 and its clinical implications. Prz Menopauzalny.
2017; 16(3): 107-111, doi: 10.5114/pm.2017.70589, indexed in Pubmed: 29507578.

23. Parasar P, Ozcan P, Terry KL. Endometriosis: epidemiology, diagnosis and clinical management. Curr Obstet Gynecol Rep. 2017; 6(1): 34-41, doi: 10.1007/s13669-017-0187-1, indexed in Pubmed: 29276652.

24. Koike N, Tsunemi T, Uekuri $\mathrm{C}$, et al. Pathogenesis and malignant transformation of adenomyosis (review). Oncol Rep. 2013; 29(3): 861-867, doi: 10.3892/or.2012.2184, indexed in Pubmed: 23242072.

25. Kucera E, Hejda V, Dankovcik R, et al. Malignant changes in adenomyosis in patients with endometrioid adenocarcinoma. Eur J Gynaecol Oncol. 2011; 32(2): 182-184, indexed in Pubmed: 21614909.

26. Aslan K, Sarı ME, Yalçın HR, et al. Coexistence of uterine adenomyosis is not associated with a better prognosis in endometrioid-type endometrial cancer. Ir J Med Sci. 2020; 189(3): 835-842, doi: 10.1007/s11845020-02172-z, indexed in Pubmed: 31970616.

27. Chao X, Wu M, Ma S, et al. The clinicopathological characteristics and survival outcomes of endometrial carcinoma coexisting with or arising in adenomyosis: A pilot study. Sci Rep. 2020; 10(1): 5984, doi: 10.1038/s41598-020-63065-w, indexed in Pubmed: 32249826.

28. Raffone A, Seracchioli R, Raimondo D, et al. Prevalence of adenomyosis in endometrial cancer patients: a systematic review and meta-analysis. Arch Gynecol Obstet. 2021; 303(1): 47-53, doi: 10.1007/s00404-02005840-8, indexed in Pubmed: 33098006.

29. Koshiyama M, Suzuki A, Ozawa M, et al. Adenocarcinomas arising from uterine adenomyosis: a report of four cases. Int J Gynecol Pathol. 2002; 21(3): 239-245, doi: 10.1097/00004347-200207000-00006, indexed in Pubmed: 12068169.

30. Siegel RL, Miller KD, Jemal A. Cancer statistics, 2018. CA Cancer J Clin. 2018; 68(1): 7-30, doi: 10.3322/caac.21442, indexed in Pubmed: 29313949.

31. Shen F, Liu Y, Lin $L$, et al. Association of benign gynaecological diseases and risk of endometrial and ovarian cancers. J Cancer. 2020; 11(11): 3186-3191, doi: 10.7150/jca.39626, indexed in Pubmed: 32231723.

32. Hever A, Roth RB, Hevezi PA, et al. Molecular characterization of human adenomyosis. Mol Hum Reprod. 2006; 12(12): 737-748, doi: 10.1093/molehr/gal076, indexed in Pubmed: 17020905.

33. $\mathrm{Hu} \mathrm{H}, \mathrm{Li} \mathrm{H}, \mathrm{He}$ Y. MicroRNA-17 downregulates expression of the PTEN gene to promote the occurrence and development of adenomyosis. Exp Ther Med. 2017; 14(4): 3805-3811, doi: 10.3892/etm.2017.5013, indexed in Pubmed: 29042983.

34. Mutter GL, Lin MC, Fitzgerald JT, et al. Altered PTEN expression as a diagnostic marker for the earliest endometrial precancers. J Natl Cancer Inst. 2000; 92(11): 924-930, doi: 10.1093/jnci/92.11.924, indexed in Pubmed: 10841828.

35. Rafnar T, Gunnarsson B, Stefansson OA, et al. Variants associating with uterine leiomyoma highlight genetic background shared by various cancers and hormone-related traits. Nat Commun. 2018; 9(1): 3636, doi: 10.1038/s41467-018-05428-6, indexed in Pubmed: 30194396. 